\title{
VUS-type alteration in POLD1 and microsatellite instability in a metastatic luminal B breast cancer patient
}

\author{
Catarina Marchon da Silva ${ }^{1}$, Denis Shimba², Denise Oishi², Allyne Cagnacci², Ana Carolina Ribeiro Chaves de Gouvea ${ }^{2}$ and Felipe Ades ${ }^{2}$ \\ ${ }^{1}$ Faculdade de Medicina, Universidade Municipal de São Caetano do Sul, São Caetano do Sul, SP 09521-160, Brazil \\ ${ }^{2}$ Centro Especializado em Oncologia, Hospital Alemão Oswaldo Cruz, São Paulo, SP 01323-020, Brazil
}

\begin{abstract}
Microsatellite instability (MSI) and POLD1 mutations are usually described in colorectal tumours in patients with polyposis syndrome but rarely found in breast tumours. This case describes a metastatic luminal $B$ breast tumour in a young patient with an important family history of cancer. Mutational studies found a Variant of Uncertain Significance (VUS)-type alteration in POLD1 that motivated the study for MSI, which was found positive. Recent data point towards the use of pembrolizumab as a treatment option for tumour presenting with MSI instead of chemotherapy.
\end{abstract}

Keywords: POLD1, luminal B breast cancer, pembrolizumab, microsatellite instability

\section{Introduction}

Breast cancer is a heterogeneous disease with high prevalence in developed and developing countries. Breast cancer has its highest incidence in women between 40 and 70 years old, affecting around 2 million women in 2018 [1]. The disease is divided into four main immunohistochemical profiles, according to receptor expression for oestrogen (ER), progesterone (PgR) and human epidermal 2 growth factor gene receptor (HER2): Iuminal A, luminal B, Triple-negative and HER2 positive [2]. As a result, treatment is more individualised depending on the subtype.

Microsatellites are nucleotide repetitions of the DNA, responsible for genomic maintenance. During replication, they may suffer errors which are quickly repaired, and normal replication follows. Hence, microsatellite instability (MSI) is the name given to the germline allele portion of the microsatellite that has suffered addition or deletion of its units, product of loss of cell capacity to correct errors associated with replication, resulting in a somatic length alteration $[3,11]$.

Tumours presenting MSI have higher mutation rates since DNA-repairing genes are either mutated or inactive, resulting in constant DNA exposure to transformation during cell replication. This process leads to neoantigen synthesis, allowing easier recognition by the immune system. Since immunotherapy aims to amplify the immune system while down-regulating tumour-immune evasion mechanisms, it is a useful tool in tumours with these characteristics.

Correspondence to: Felipe Ades

Email: felipeades@gmail.com

ecancer 2020, 14:1002

https://doi.org/10.3332/ecancer.2020.1002

Published: $21 / 01 / 2020$

Received: 26/08/2019

Publication costs for this article were supported by ecancer (UK Charity number 1176307).

Copyright: $($ ) the authors; licensee ecancermedicalscience. This is an Open Access article distributed under the terms of the Creative Commons Attribution License (http:// creativecommons.org/licenses/by/3.0), which permits unrestricted use, distribution, and reproduction in any medium, provided the original work is properly cited. 
In this scenario, pembrolizumab, an anti-PD-1 monoclonal antibody, prevents lymphocyte down-regulation after tumour PD-L1 and lymphocyte PD-1 binding [4]. As a result, tumour proteins are better recognised for increasing the immune system efficacy and causing cytotoxic cell death. A recent study demonstrated that tumours presenting MSI, regardless of tumour etiology, can be treated with pembrolizumab.

POLD1 is a gene that codes the delta variation of the DNA polymerase, responsible for DNA-associated repair through base excision during cell replication. Its mutation is more typically described in colorectal tumours with MSI, usually in familiar polyposis syndrome patients [5].

As discussed earlier, MSI usually presents itself alongside deficient, mutated or inactive DNA-repair genes; as POLD1 genes code q-repairing enzymes, mutations in this particular gene could be found in MSI tumours. This correlation could explain high rates of tumour mutation and pembrolizumab response rates during treatment regime, as biological and scientific rationale infers that the number of neoantigens in a tumour with deficient q-repair genes and MSI profile would allow amplified immune response-due to immunotherapy-to interfere greatly with cellular proliferation and macroscopically, disease progression.

\section{Case report}

A 42-year-old female patient, G. C. C, no comorbidities, reporting important breast cancer family history, with four first-degree relatives diagnosed with breast cancer under 40 years old. She was diagnosed with breast malignant neoplasm during her pregnancy, undergoing adenomastectomy and sentinel lymph node dissection. The anatomopathological study revealed a grade 3 ductal invasive carcinoma, measuring $0.8 \mathrm{~cm}$ with a negative sentinel lymph node. Immunohistochemical profile was ER 85\%, PgR (-), HER2 (-), Ki-67 70\%, characterising a luminal B tumour (Table 1). At the time of the diagnosis, the patient was advised to undergo genetic testing for BRCA1 and BRCA2 genes, which did not reveal any mutations. She decided not to take adjuvant tamoxifen, radiotherapy nor chemotherapy since she was pregnant at the time of the diagnosis.

Table 1. Tumour immunohistochemistry illustrating a luminal B tumour phenotype.

\begin{tabular}{|c|c|c|}
\hline \multicolumn{3}{|c|}{ Left Breast } \\
\hline Antibody & Clone & Interpretation \\
\hline EGFR & $31 \mathrm{G7}$ & Negative in the tumor cells \\
\hline Progesterone Receptor (PR) & PgR636 & Negative in the tumor cells \\
\hline Estrogen Receptor (ER) & SP1 & Positive in $85 \%$ of the tumor cells \\
\hline c-erB-2 & SP3 & Escore 0 \\
\hline Ki-67 & MIB-1 & Positive in $70 \%$ of the tumor cells \\
\hline CK5 & Polyclonal & Negative in the tumor cells \\
\hline Antigen & & Result \\
\hline Estrogen Receptor & \multicolumn{2}{|c|}{ Positive (moderate intensity, $70 \%$ of the cells) } \\
\hline Progesterone Receptor & \multicolumn{2}{|l|}{ Negative } \\
\hline HER-2 & \multicolumn{2}{|l|}{ Negative } \\
\hline Ki-67 (MIB-1) & \multicolumn{2}{|c|}{ Positive (30\%) } \\
\hline Cytokeratin 5 & \multicolumn{2}{|l|}{ Negative } \\
\hline TTF-1 & \multicolumn{2}{|l|}{ Negative } \\
\hline Cytokeratin 7 & \multicolumn{2}{|l|}{ Positive } \\
\hline Cytokeratin 20 & \multicolumn{2}{|l|}{ Negative } \\
\hline Mammaglobin & \multicolumn{2}{|l|}{ Negative } \\
\hline
\end{tabular}


After breastfeeding, she started her treatment with tamoxifen $20 \mathrm{mg} /$ day for 2 years, when she presented disease progression in the sternum body. She was then treated with goserelin, zoledronic acid and exemestane. In addition, she received radiotherapy in the sternum region, as this was her only metastatic lesion. She had stable disease for 3 years when a PET-CT scan showed increased FDG-uptake in the same region of the sternum body (Figures 1 and 2). She was treated locally with cryotherapy and continued with the same systemic regimen. After another 2 years of disease control, a new PET-CT scan shown mixed-pattern lesions on the upper half of the sternum body with bone cortical erosion and small soft-tissue component in the anterior and lateral margins, measuring $5 \mathrm{~cm}$ with standarized uptake value (SUV) $=4.4$. Physical examination exhibited a painful lump in the sternum bone, compatible with the PET/CT image. Treatment was then changed to intramuscular fulvestrant $500 \mathrm{mg}$ + leuprorelin every 28 days, with complete shrinkage of the sternum lump and symptom improvement. The patient has now stable disease for almost 2 years since starting this treatment line.

Due to important family history and young age at the time of diagnosis, a full germline mutation panel was ordered to investigate further mutations associated. The panel detected for a VUS-type alteration in POLD1 gene - c.1923dupC (p.Thr642Hisfs*97) - kind of mutation is possibly associated with MSI. As high expression of POLD1 is associated with poor prognosis [6], and there are therapeutic implications to this, tumoural fragments from the primary tumour were then tested by immunohistochemistry for mismatch repair (MMR) deficiencies, which revealed a MSH6 instability, characterising MSI phenotype (Table 2). These recent findings may explain the indolent curse of the disease and open the possibility of treatment with immunotherapy in subsequent lines of treatment.

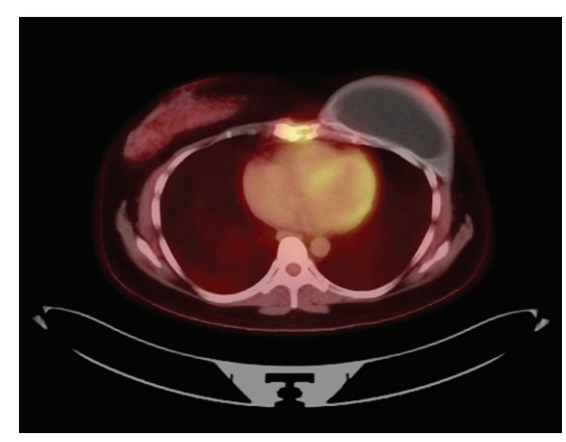

Figure 1. Patient's PET Scan from 21 November 2019. Axial plane, revealing disease in sternum body.

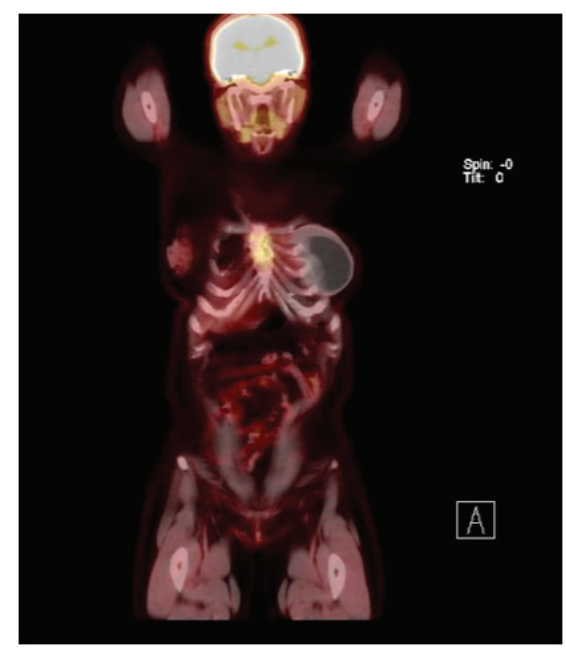

Figure 2. Patient's PET Scan from 21 November 2019. Sagittal plane, revealing disease in sternum body. 
Table 2. Immunohistochemistry revealing MMR instability in MSH6, characterizing MSI phenotype. Result issued on 27 March 2019.

\begin{tabular}{|l|l|}
\hline \multicolumn{2}{|c|}{ Antibodies } \\
\hline MLH1 & Nuclear reaction preserved = 'stable' profile \\
\hline MSH2 & Nuclear reaction preserved = 'stable' profile \\
\hline MSH6 & Loss of reaction = 'unstable' profile \\
\hline PMS2 & Nuclear reaction preserved = 'stable' profile \\
\hline
\end{tabular}

\section{Discussion}

This is a luminal B breast cancer case with a typical presentation at diagnosis in which mutation testing broadened the possibilities of treatment options. POLD1 germline mutations are rare in breast cancer, as it is more commonly found in colorectal cancer patients with polyposis syndrome [5].

Interestingly, POLD1 mutations are described interacting with BRCA1 and BRCA2 genes [7], increasing the risk for allelic mutations, leading to increased chances of tumour development, which was not seen in the previously described case. Furthermore, the presence of MSI, associated with POLD1 alterations or without is rarely described in breast malignant tumours [8], and in the case of luminal tumours it was previously described with lower overall survival and worst prognosis [9].

Tumours with MSI usually present a high tumoural mutational burden, often associated with the presence of neoantigens and higher response to treatment with immunotherapy. The use of pembrolizumab was tested in a phase II study including 41 patients with colon cancer with or without MSI and non-colorectal cancer with MSI. The response rate and progression-free survival (PFS) rate was 40\% and 78\% in the deficient cohort and $0 \%$ and $11 \%$ in the proficient cohort. The median PFS and median overall survival were not reached in the deficient cohort and 2.2 and 5.0 months in the proficient cohort. Whole-exome sequencing revealed a mean 1,782 somatic mutations per tumour in the MSI cohort and 73 mutation in the proficient cohort. High somatic mutations were associated with longer PFS [9].

This is a rare case of VUS-type alteration in POLD1 and MSI in breast cancer. The discovery of the VUS-type alteration partnered with the investigation for MSI opens new treatment opportunities for this patient. In the future, molecular testing will allow better understanding of disease biology and tailor therapy for each specific individual.

The complexity of interactions between somatic and germline mutations in the carcinogenic behaviour of cancer and disease heterogeneity poses a challenge to the future investigation of cancer treatment. However, with the accumulating real life and study data added to translational and preclinical studies on cancer heterogeneity, we are starting to unveil some of the mechanism and pave the way towards more personalised medicine.

\section{Conclusion}

Finally, the use of pembrolizumab on solid tumours with MSI leads to better tumour response and control if not complete response [4] and could be considered as an option for patients who are of young age and with tumours displaying such characteristics. The medication may be able to stop disease progression while maintaining quality of life and presenting fewer side effects [11].

Since there are few described cases of POLD1 mutation and MSI in breast cancer, pembrolizumab may be one more treatment option for the reported patient, reserving the use of chemotherapy for subsequent treatment lines, if needed. Despite these findings and new possibilities, more studies focusing on the relationship between the use of pembrolizumab in tumours with POLD1 mutation and MSI in breast cancer, in general, are required. 


\section{Conflicts of interest}

The authors have declared no relevant conflicts of interest related to this work.

\section{Funding declaration}

The authors have received no funds for the development of this work.

\section{References}

1. International Agency for Research on Cancer, World Health Organization (2019) Estimated number of new cases in 2018, worldwide, both sexes, all ages. GLOBOCAN

2. Sarturi PR, Ademar DC Jr, and de Morais CF (2012) Perfil imunohistoquímicodo câncer de mama de pacientes atendidas no Hospital do Câncer de Cascavel-Paraná Rev Bras Onco Clínica. 8(29) 121-124

3. Lopez G, Corti C, and Pesenti C, et al (2018) Mismatch repair protein loss as a prognostic and predictive biomarker in breast cancers regardless of microsatellite instability JNCl Cancer Spectrum 2(4) pky056 https://doi.org/10.1093/jncics/pky056

4. Le DT, Uram JN, and Wang H, et al (2015) PD-1 blockade in tumors with mismatch-repair deficiency N Engl J Med 372(26) 2509-2520 https://doi.org/10.1056/NEJMoa1500596 PMID: 26028255 PMCID: 4481136

5. Sigurdson AJ, Hauptmann M, and Chatterjee N, et al (2004) Kin-cohort estimates for familial breast cancer risk in relation to variants in DNA base excision repair, BRCA1 interacting and growth factor genes BMC Cancer 4(1) 9 https://doi.org/10.1186/1471-2407-4-9 PMID: 15113441 PMCID: 408462

6. Qin Q, Tan Q, and Li J, et al (2018) Elevated expression of POLD1 is associated with poor prognosis in breast cancer Oncol Lett https:// doi.org/10.3892/ol.2018.9392 PMID: 30344713 PMCID: 6176253

7. Valle L, Hernández-Illán E, and Bellido F, et al (2014) New insights into POLE and POLD1 germline mutations in familial colorectal cancer and polyposis Hum Mol Genet 23(13) 3506-3512 https://doi.org/10.1093/hmg/ddu058 PMID: 24501277

8. Anbazhagan R, Fujii H, and Gabrielson E (1999) Microsatellite instability is uncommon in breast cancer Clin Cancer Res 5(4) 839-844 PMID: 10213220

9. de la Chapelle A (2003) Microsatellite Instability N Engl J Med 349(3) 209-210 https://doi.org/10.1056/NEJMp038099 PMID: 12867603

10. Wang F, Zhao Q, and Wang Y-N, et al (2019) Evaluation of POLE and POLD1 mutations as biomarkers for immunotherapy outcomes across multiple cancer types JAMA Oncol https://doi.org/10.1001/jamaoncol.2019.2963

11. University of Pittsburgh Schools of the Health Sciences (2016) Immunotherapy improves survival, quality of life in rapidly progressing head and neck cancer ScienceDaily [www.sciencedaily.com/releases/2016/06/160606135603.htm]

12. Jansen AM, van Wezel T, and van den Akker BE, et al (2016) Combined mismatch repair and POLE/POLD1 defects explain unresolved suspected Lynch syndrome cancers Eur J Hum Genet 24(7) 1089-1092 https://doi.org/10.1038/ejhg.2015.252 PMCID: 5070903 